\title{
Circular RNA ERBB2 Contributes to Proliferation and Migration of Airway Smooth Muscle Cells via miR-98-5p/IGFIR Signaling in Asthma
}

\author{
Jun-Qian Huang' \\ Fang Wang ${ }^{2}$ \\ Long-Tao Wang ${ }^{3}$ \\ Yong-Mei $\mathrm{Li}^{4}$ \\ Jun-Li Lu \\ Jian-You Chen ${ }^{2}$
}

'Department of Respiratory and Critical Medicine, Qingdao Chengyang District People's Hospital, Qingdao, Shandong, People's Republic of China; ${ }^{2}$ Department of Respiratory Medicine, Qingdao Municipal Hospital, Qingdao, Shandong, People's Republic of China; ${ }^{3}$ Department of Critical Care Medicine, Qingdao Chengyang District People's Hospital, Qingdao, Shandong, People's Republic of China; ${ }^{4}$ Department of Clinical Pharmacy, Qingdao Chengyang District People's Hospital, Qingdao, Shandong, People's Republic of China; ${ }^{5}$ Qingdao Chengyang District People's Hospital, Qingdao, Shandong, People's Republic of China
Background: Asthma belongs to chronic inflammatory respiratory diseases characterized by airway inflammation and remodeling. Circular RNAs (circRNAs) are promising therapeutic targets for various diseases, including asthma. In this work, we aim to investigate the role of circular RNA Erb-B2 receptor tyrosine kinase 2 (circERBB2) during progression of asthma. Methods: Human airway smooth muscle cells (ASMCs) were treated with platelet-derived growth factor BB (PDGF-BB) to mimic cell remodeling. The expression of circERBB2, microRNA-98-5p (miR-98-5p), and insulin-like growth factor 1 receptor (IGF1R) was measured by qRT-PCR. Cell proliferation, migration and apoptosis were determined by cell counting-8 (CCK-8), transwell, and flow cytometry. Protein levels of PCNA, MMP-9, IGF1R were evaluated using Western blotting. The levels of tumor necrosis factor- $\alpha$ (TNF- $\alpha$ ), interleukin-1 $\beta$ (IL-1 $\beta$ ), and IL-6 were detected by enzyme-linked immunosorbent assay (ELISA). Luciferase reporter gene experiment was adopted to evaluate the targeting relationship between miR-98-5p with $\operatorname{circERBB2}$ and IGF1R. Interaction between RNAs was determined by RNA pulldown and RIP assay.

Results: The depletion of $\operatorname{circERBB2}$ attenuated the proliferation, migration, and levels of inflammatory factors induced by PDGF-BB and cell apoptosis. CircERBB2 was identified to directly interact with $m i R-98-5 \mathrm{p}$, and overexpression of $m i R-98-5 p$ abolished the function of circERBB2 on PDGF-BB-stimulated ASMCs. IGF1R was identified as a target of $m i R-98-5 p$, and knockdown of $I G F 1 R$ relieved the PDGF-BB-induced ASMCs proliferation and migration.

Conclusion: Our work disclosed that knockdown of circERBB2 suppressed PDGF-BBcaused proliferation, migration and inflammatory response of ASMCs, through regulating miR-98-5p/IGF1R signaling, presented circERBB2 as a promising therapeutic target for asthma.

Keywords: asthma, circERBB2, miR-98-5p, IGF1R, proliferation, migration

\section{Introduction}

Asthma belongs to chronic inflammatory respiratory diseases, which frequently occurs in children. ${ }^{1-3}$ The increased incidence of asthma has become a severe public health issue. ${ }^{2}$ Asthma is commonly featured by airway inflammation, remodeling and hyperresponsiveness of the airway. ${ }^{4,5}$ Airway inflammation is the most important pathological process during asthma, which is caused by infiltration of several cell types including neutrophils, mast cells, eosinophils and Th2 cells, and accompanied with elevated secretion of inflammatory factors, such as tumor
Correspondence: Jian-You Chen Department of Respiratory Medicine, Qingdao Municipal Hospital, Qingdao, Shandong, People's Republic of China Email youchanmou9834@I63.com 
necrosis factor- $\alpha$ (TNF- $\alpha$ ), interleukin-6 (IL-6) and IL$1 \beta .^{6,7}$ Anti-inflammatory medicines and bronchodilators are commonly used for managing inflammation and hyperresponsiveness of the airway, ${ }^{5}$ whereas airway remodeling remains a challenge for asthma treatment. Platelet-derived growth factor BB (PDGF-BB), significantly upregulated in asthmatic tissues, has been widely reported to be able to induce ASMC proliferation and migration and exacerbate the airway remodeling. ${ }^{8-10}$ Understanding the mechanisms involved in airway damage, especially the airway inflammation and remodeling, is therefore important for discovering effective therapeutic approaches for asthma.

Insulin-like growth factor 1 receptor (IGF1R) is a tyrosine kinase that is widely expressed in cells and plays a central role in the IGF signaling transduction, which is closely associated with survival, proliferation, differentiation, and metastasis of cells. ${ }^{11}$ More and more research has implied the participation of IGF signaling in abnormal airway functions. For example, it was indicated that IGF1 and IGFBP3 were involved in airway inflammation and remodeling during allergic response. ${ }^{12,13}$ Upregulated level of IGF1R was spotted in bronchoalveolar lavage cells of patients with asthma. ${ }^{14}$ A recent study revealed that IGF1R deficient mice exhibited reduced allergic airway inflammation. ${ }^{15}$ Therefore, targeting IGF1R may be a novel and effective approach for asthma therapy.

Circular RNAs (circRNAs) are noncoding RNAs that are characterized by covalently closed RNA structure. ${ }^{16}$ In recent years, mounting evidence has been revealing the important role of circRNAs during various pathological processes, especially in cancers. ${ }^{17}$ Yet, the functions of circRNA during asthma progression are largely unknown. Erb-B2 receptor tyrosine kinase 2 (ERBB2, also known as HER2) is one of the most studied oncogenes, being considered a breast cancer biomarker located on chromosome 17q12. ERBB2 serves as a tyrosine kinase receptor. ${ }^{18,19}$ CircERBB2 is a circRNA derived from ERBB2 gene locus, which is implicated as a tumor activator in several cancers. ${ }^{20}$ Huang and colleagues firstly identified that circERBB2 is located in the nucleus to facilitate the function of RNA polymerase, leading to activated proliferation and progression of gastric cancer cells. ${ }^{20}$ Further study even proposed circERBB2 as a prognostic biomarker for gastric cancer. ${ }^{21}$ It is well recognized that circRNAs usually directly interact with microRNAs (miRNAs) and proteins to regulate gene expression. ${ }^{17}$ MiRNAs are short sequenced noncoding RNAs that bind with the $3^{\prime}$ UTR regions of targeted mRNAs to suppress transcription or accelerate RNA degradation. ${ }^{22}$ Gao et al indicated that circERBB2 acted as a sponge of miR-7-5p to release the expression of downstream target FOXM1, which facilitated the development of lung cancer. ${ }^{23}$ MiR-98-5p was proposed as a potential diagnostic biomarker for bronchial asthma in children, and affected the level of IL-13 in serum. ${ }^{24}$ However, its detailed functions in asthma still need profound studies. Meanwhile, the interaction between circERBB2 and miR-98-5p has not been reported previously.

In this study, we aimed to discuss the role of circERBB2 during airway inflammation in asthma, defining the possible regulatory mechanisms that involve the miR-98-5p and IGF1R, provided promising targets and diagnostic biomarkers for asthma.

\section{Materials and Methods Biopsy Sample Collection}

The samples of patients with asthma $(n=45)$ who had undergone fiberoptic bronchoscopy were obtained during a bronchial biopsy, and samples from the healthy volunteers $(n=45)$ with no disease. ${ }^{25-27}$ The experiments were performed following the guidance of Qingdao Municipal Hospital and in accordance with the Declaration of Helsinki. All patients have signed the informed consents. The studies involving human participants were reviewed and ethically approved by Qingdao Municipal Hospital (Approval No. 2021081103).

\section{Cell Culture and Treatment}

Human ASMCs were purchased from Sciencell (USA) and cultured in high glucose-DMEM (Invitrogen, USA) supplemented with $10 \%$ fetal bovine serum (FBS, Gibco), 100 $\mu \mathrm{g} / \mathrm{mL}$ streptomycin and $100 \mathrm{U} / \mathrm{mL}$ penicillin (Sigma, USA) in a humidified $37^{\circ} \mathrm{C}$ incubator filled with $5 \%$ $\mathrm{CO}_{2}$. The platelet-derived growth factor BB (PDGF-BB) was reported to be capable of stimulating the airway inflammation and remodeling. To establish the cell model of asthma, ASMCs were deprived of FBS for 24 hours, followed by administration of PDGF-BB at a final concentration of $25 \mathrm{ng} / \mathrm{mL}$.

\section{Cell Transfection}

Small hairpin RNAs targeting circERBB2 (shcircERBB2-1 and shcircERBB2-2) and IGFIR (shIGF1R-1 and shIGF1R1 ), overexpressing plasmids inserted with $\operatorname{circERBB2}$ (pciR- 
circERBB2) and IGF1R (pcDNA-IGF1R) were full-length synthesized and obtained (GenScript, China). MiR-98-5p mimics and inhibitors were purchased from RiboBio (China). For cell transfection, oligonucleotides $(1 \mu \mathrm{g})$ were mixed with Lipofectamine 2000 (Invitrogen) in line with manufacturer's instruction.

\section{Quantitative Real-Time PCR}

Total RNA of the samples and cells were extracted by using Trizol reagent (Thermo, USA), transcribed to cDNAs by using PrimeScript RT reagent kit (Takara, Japan), and then quantified by using SYBR Green Master kit (Applied Biosystems, USA). The relative levels of IGF1R, circERBB2, and miR-98-5p were normalized to GAPDH and U6 snRNA using a $2^{-\Delta \Delta \mathrm{Ct}}$ method. The primers were as following:

IGF1R, sense, 5'-TCGACATCCGCAACGACTATC3', and antisense, 5'- CCAGGGCGTAGTTGTAGAAGAG-3';

circERBB2, sense, 5'-GCCCTGGTCACCTACAACA C-3', and antisense, 5'-TGGATATCAGGGACAGGCAG T-3';

miR-98-5p, sense, 5'-CACCGCA GAAGCGGCACTT TATAAGCGAACT-3', and antisense, 5'-TTATAAAGTGC CGC TTCTGCTTATAAGTTCGC-3';

GAPDH, sense, 5'-GGGCTCATCTGAAGGGTGGTG CTA-3', and antisense, 5'-GTGGGGGAGACAGAAGGG AACAGA-3';

U6, sense, 5'-CTCGCTTCGGCAGCACATA-3', and antisense, 5'-AACGCTTCACGAATTTGCGT-3'.

\section{Western Blotting Assay}

ASMCs were homogenized using RIPA lysis buffer (Thermo) to obtain total proteins. The proteins were then quantified and separated on $10 \%$ SDS-PAGE gel, shifted to polyvinylidene difluoride (PVDF) membranes (Millipore, USA), blocked in 5\% skim milk, hatched in specific primary antibodies including IGF1R (1:2000, Santa Cruz, USA), MMP-9 (1:1000, Santa Cruz), MMP-9 (1:1000, Santa Cruz, USA), Bax (1:1000, Abcam, USA), Bcl-2 (1:1000, Abcam, USA), cleaved caspase-3 (1:1000, Abcam, USA), and GAPDH (1:1000, Proteintech, China) at $4^{\circ} \mathrm{C}$ overnight. Next day, the membranes were visualized after incubation with HRP-conjugated secondary antibodies (Proteintech, China) and ECL solution (Millipore).

\section{Cell Viability, Migration and Apoptosis}

Cell viability was detected by using cell countin-8 kit (CCK8 , Beyotime, China). In short, cells were seeded in 96-well plates with 5,000 cells per well. At the indicated time points, CCK-8 solution (10 $\mu \mathrm{L})$ was added into each well and incubated for extra 2 hours. Then, the absorbance values at $450 \mathrm{~nm}$ were detected by a spectrophotometer (BD Biosciences, USA). For cell migration, cells were suspended in FBS-free medium, and seeded in the upper chambers of Transwell plates (Corning, USA), while the lower chambers were filled with a complete medium. The upper chambers were collected after incubation for 48 hours, washed in PBS, fixed in methanol, and dyed with $1 \%$ crystal violet. The migrated cells were then counted and calculated. Cell apoptosis was measured by using a FITC-Annexin V/PI detection kit following manufacturer's protocol.

\section{Enzyme-Linked Immuno Sorbent Assay (ELISA)}

The release of inflammatory factors, IL-1 $\beta$, TNF- $\alpha$, IL- 6 , and IL-13 by ASMCs were determined by commercial ELISA kits (Beyotime) in accordance with manufacturer's instruction.

\section{Luciferase Reporter Assay}

The potential binding sites of miR-98-5p on circERBB2 and the 3'-UTR of FGF1 were predicted on ENCORI website. The wild type (WT) and mutated (MUT) sequences of circERBB2 and the 3'-UTR of FGF1 were inserted into pGL3-control plasmids, which were full-length synthesized and obtained (GenScript, China). Cells were transfected with the constructed vectors and miR-98-5p mimics, along with the pRL-TK as internal control. The luciferase activity was determined after 48-hour incubation with a dual luciferase reporter assay system (Promega).

\section{RNA Pulldown}

For miR-98-5p pulldown circRNAs, the biotin-labelled wild sequence (WT) and mutated sequence (MUT) were purchased from RiboBio and linked with magnetic beads (Invitrogen). Cells were lysed, sonicated, and centrifuged to collect the supernatant. The lysates were then incubated with the labelled beads for 6 hours at $4^{\circ} \mathrm{C}$, then washed with PBS, and quantified by qRT-PCR.

\section{RNA Immunoprecipitation}

Magna RIP RNA-binding protein immunoprecipitation kit (Millipore) was used for RIP assay in accordance with manufacturer's instruction. In short, cells lysates were obtained by using lysis buffer and sonication, then incubated with Ago2 antibody or IgG antibody-conjugated 
beads for 3 hours at room temperature. The precipitation was washed and eluted, the enriched circERBB2 and miR$98-5 \mathrm{p}$ were analyzed by qRT-PCR assay.

\section{Statistical Analysis}

Data were expressed as mean \pm SD and analyzed by using SPSS 20.0 software. Comparison between two or multiple groups was determined by student's $t$ test or one-way ANOVA analysis, respectively. $p<0.05$ was considered as statistically significant.

\section{Results}

The Expression of circERBB2 Was Upregulated in the Biopsy Samples of Patients with Asthma and PDGF-BB-

\section{Stimulated ASMCs}

We first determined whether circERBB2 expression was associated with asthma. Analysis of clinical biopsy samples indicated that expression of circERBB2 was notably higher in biopsy samples collected from asthma patients, compared with that of healthy donors (Figure 1A). In addition, the PDGF-BB-stimulated ASMCs expressed elevated levels of circERBB2 and $I G F 1 R$, whereas decreased level of miR-98-5p, in comparison with the unstimulated group (Figure 1B-D).

\section{CircERBB2 Knockdown Suppressed PDGF-BB-Stimulated Proliferation and Migration of ASMCs}

To clarify the specific role of circERBB2 in ASMCs, we performed $\operatorname{circERBB2}$ knockdown experiments. Transfection of two shRNA sequences against circERBB2 could both effectively downregulate the expression of circERBB2 (Figure 2A). Here, we chose shcircERBB2-2 for the following experiments. As shown in Figure 2B and D, knockdown of circERBB2 notably suppressed PDGF-BB -stimulated proliferation and migration of ASMCs. Results

B

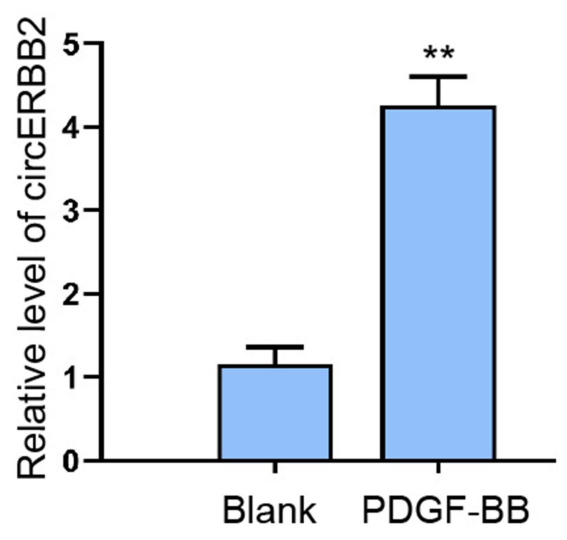

D

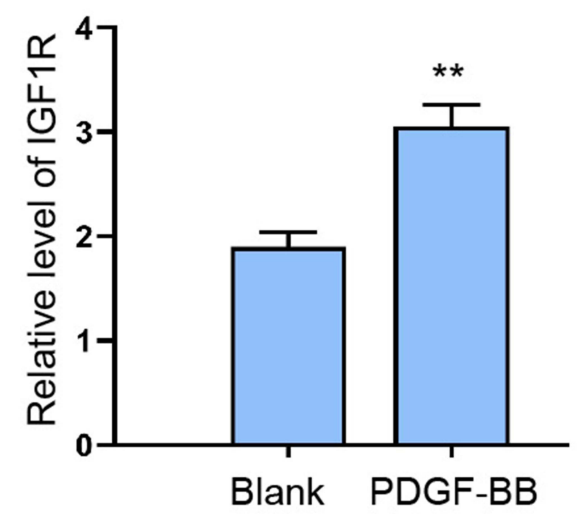

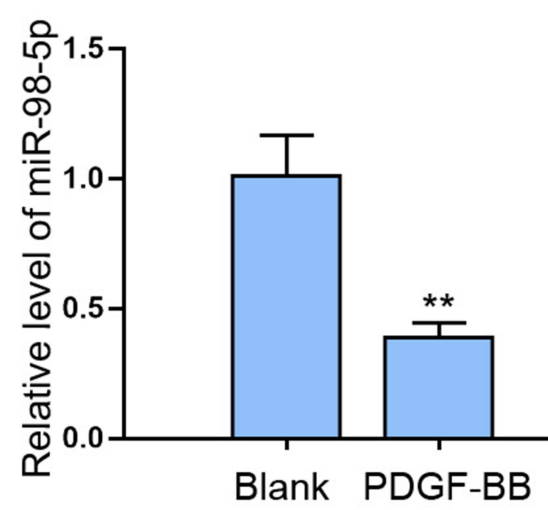

Figure I The expression of circERBB2 was upregulated in the biopsy samples of patients with asthma and PDGF-BB-stimulated ASMCs. (A) QRT-PCR quantification of circERBB2 level in biopsy samples collected from patients with asthma $(n=45)$ and healthy donors $(n=45)$. (B-D) ASMCs were stimulated with PDGF-BB $(25 \mathrm{ng} / \mathrm{mL})$ for 24 hours, the levels of circERBB2, IGFIR, and miR-98-5p were detected by qRT-PCR assay. ${ }^{*} p<0.05$, ** $p<0.01$. 

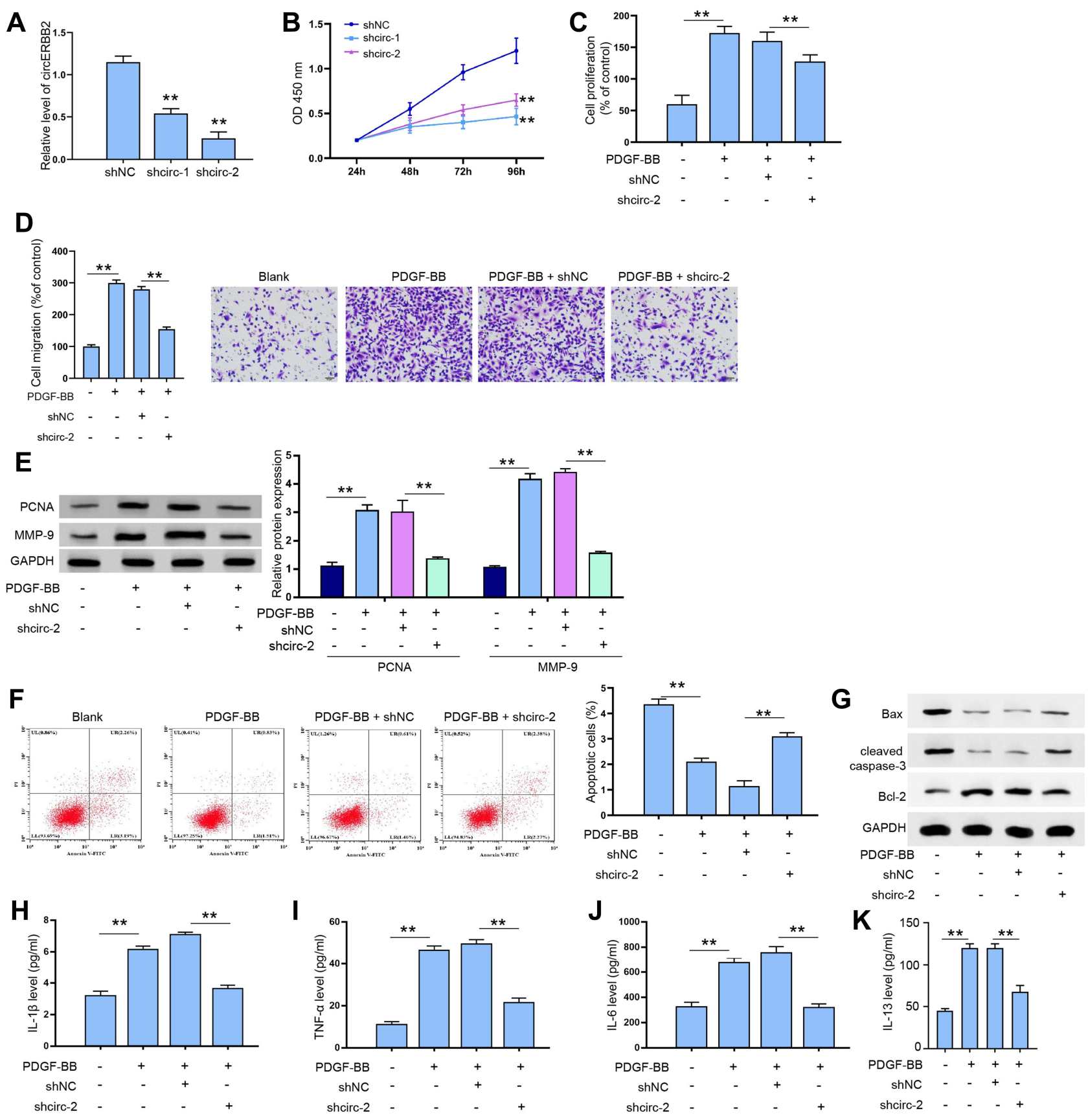

Figure 2 CircERBB2 knockdown suppressed PDGF-BB -stimulated proliferation and migration of ASMCs. (A) Expression of circERBB2 in ASMCs transfected with shcircERBB2-I, shcircERBB2-2, or negative control (shNC) were detected by qRT-PCR experiment. (B, C) ASMCs were treated with PDGF-BB and depleted of circERBB2. Cell proliferation was detected by CCK-8 assay. (D) Cell migration was detected by Transwell assay. (E) The expression of PCNA and MMP-9 were measured by Western blotting. (F) Apoptosis of ASMCs was measured by flow cytometry. (G) The expression of Bax, cleaved caspase-3, and Bcl-2 was measured by Western blot analysis. (H, K) The secreted levels of IL-I $\beta$, IL-6, TNF- $\alpha$, and IL-I 3 were determined by ELISA assay. Shcirc-I, shcircERBB2-I; shcirc-2, shcircERBB2-2; shNC, negative control. **p < 0.0 I.

from Western blotting further showed decreased levels of PCNA and MMP-9, the corresponding makers of proliferation and migration, after knockdown of circERBB2, comparing with ASMCs-stimulated by PDGF-BB (Figure 2E). CircERBB2 depletion also accelerated the apoptosis of ASMCs under PDGF-BB-stimulation (Figure 2F and G). Moreover, PDGF-BB stimulation elevated levels of IL-1 $\beta$,
IL-6, TNF- $\alpha$, and IL-13 and circERBB2 knockdown notably reversed this effect (Figure 2H-K).

\section{CircERBB2 Directly Interacted with miR-98-5p}

As mentioned above, we observed oppositely expressed circERBB2 and $m i R-98-5 p$ in ASMCs after PDGF-BB 
treatment. Hence, we wonder if circERBB2 functions through regulating $m i R-98-5 p$. According to the predicted results from the online website ENCORI, there existed potential binding sites between circERBB2 and miR-98-5p (Figure 3A). We constructed circERBB2 reporter gene vectors with wild type (WT) or mutated binding sequences (MUT) of miR-98-5p, and further conducted the luciferase reporter gene assay via co-transfection of the vectors and $m i R-98-5 p$ mimics. Transfection with $m i R-98-5 p$ mimics and inhibitors effectively altered the level of $m i R-98-5 p$ (Figure 3B). As shown in Figure 3C, miR-98-5p remarkably suppressed the luciferase activity of WT rather than the MUT, manifesting the potential interaction between wildtype circERBB2 and miR-98-5p. We further performed RNA pulldown and RIP assay, and the enriched levels of circERBB2 and $m i R-98-5 p$ revealed that $\operatorname{circERBB2}$ could directly interact with miR-98-5p (Figure 3D and E). Moreover, the knockdown of $\operatorname{circERBB2}$ led to a significantly decreased level of $m i R-98-5 p$ (Figure $3 F$ ). Meanwhile, the miR-98-5p expression was negatively correlated with circERBB2 in biopsy samples of patients with asthma (Figure 3G).

\section{MiR-98-5p Mediated the Regulation of circERBB2 to PDGF-BB-Stimulated Proliferation and Migration of ASMCs}

Subsequently, we determined the role of $m i R-98-5 p$ in circERBB2-regulated behaviors of ASMCs. We first demonstrated that miR-98-5p significantly suppressed ASMCs proliferation (Figure 4A). Next, we conducted transfection of miR-98-5p inhibitors, negative control, shcircERBB2, or $m i R-98-5 p$ inhibitors + shcircERBB2, and performed CCK-8, Transwell and flow cytometry. Results suggested that even though circERBB2 depletion could suppress the proliferation and migration of ASMCs and promoted cell apoptosis under PDGF-BB treatment, miR-98-5p inhibitors could effectively abolish these effects (Figure 4B-E).

\section{MiR-98-5p Targeted IGFIR to Suppress the Response of ASMCs to PDGF-BB-}

\section{Stimulation}

We next tried to clarify the relationship between $m i R-98-5 p$ and IGF1R. The potential binding of $m i R-98-5 p$ on 3 'UTR region of $I G F 1 R$ was predicted by ENCORI online tool (Figure 5A). And results from luciferase reporter gene assay revealed that $m i R-98-5 p$ could bind to the wild-type 3'UTR of $I G F 1 R$ rather than the mutated one (Figure $5 \mathrm{~B}$ ). The administration of $m i R-98-5 p$ mimics notably suppressed the expression of IGF1R (Figure 5C). To evaluate the functional role of $I G F 1 R$ in proliferation and migration of ASMCs, we performed depletion of IGFIR and subsequent CCK-8 and Transwell experiments. Depletion of IGF1R led to the downregulation of IGF1R (Figure 5D) and impeded proliferation of ASMCs (Figure 5E), as well as abolishing the stimulatory effect of PDGF-BB on proliferation and migration of ASMCs (Figure 5F-G). Moreover, results from
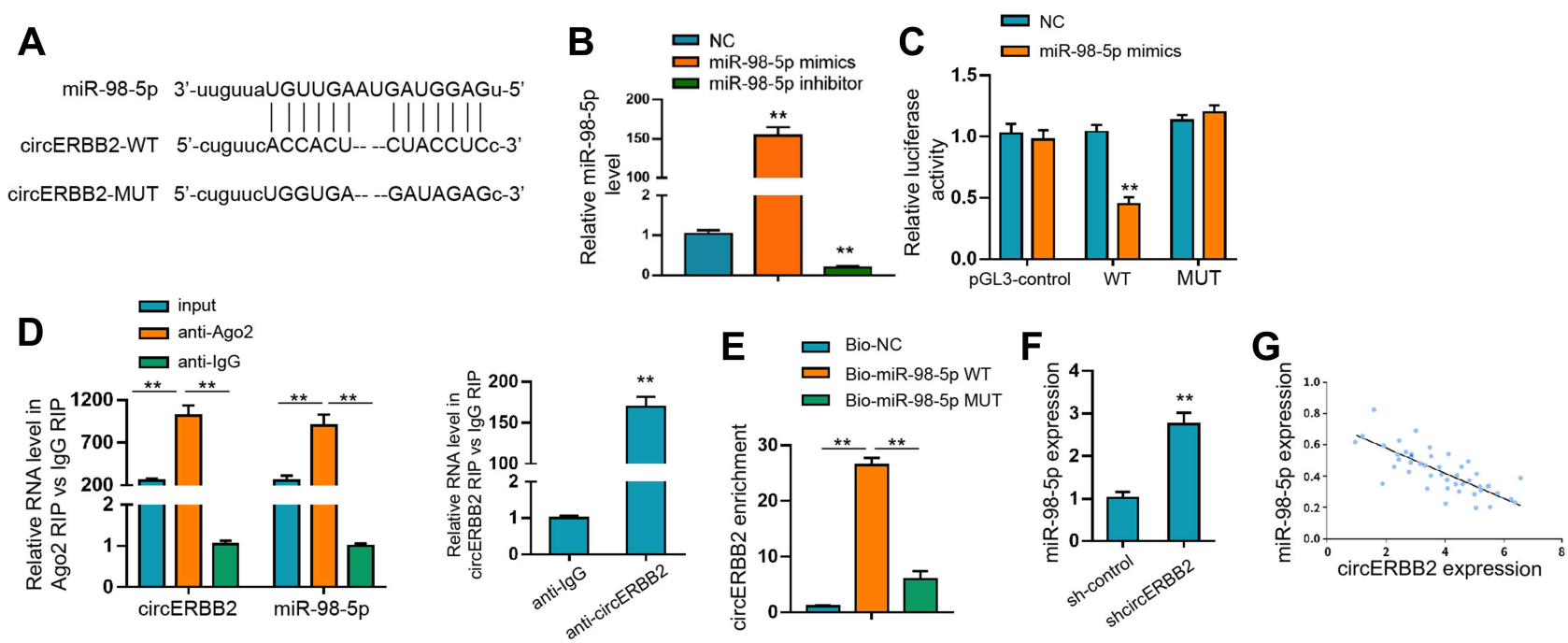

Figure 3 CircERBB2 directly interacted with miR-98-5p. (A) Predicted binding sites of miR-98-5p on circERBB2. (B) QRT-PCR assay to detect the transfection efficacy of miR-98-5p mimics in BC cells. (C) Luciferase activity of circERBB2-WT and circERBB2-MUT after transfection with miR-98-5p mimics or NC. (D, E) RNA pulldown (D) and RIP experiment (E) to evaluate the interaction between miR-98-5p and circERBB2. (F) QRT-PCR assay to measure the level of miR-98-5p in ASMCs after circERBB2 depletion. (G) QRT-PCR quantification of circERBB2 and miR-98-5p levels in biopsy samples collected from patients with asthma $(n=45)$. $* * p<0.01$. 

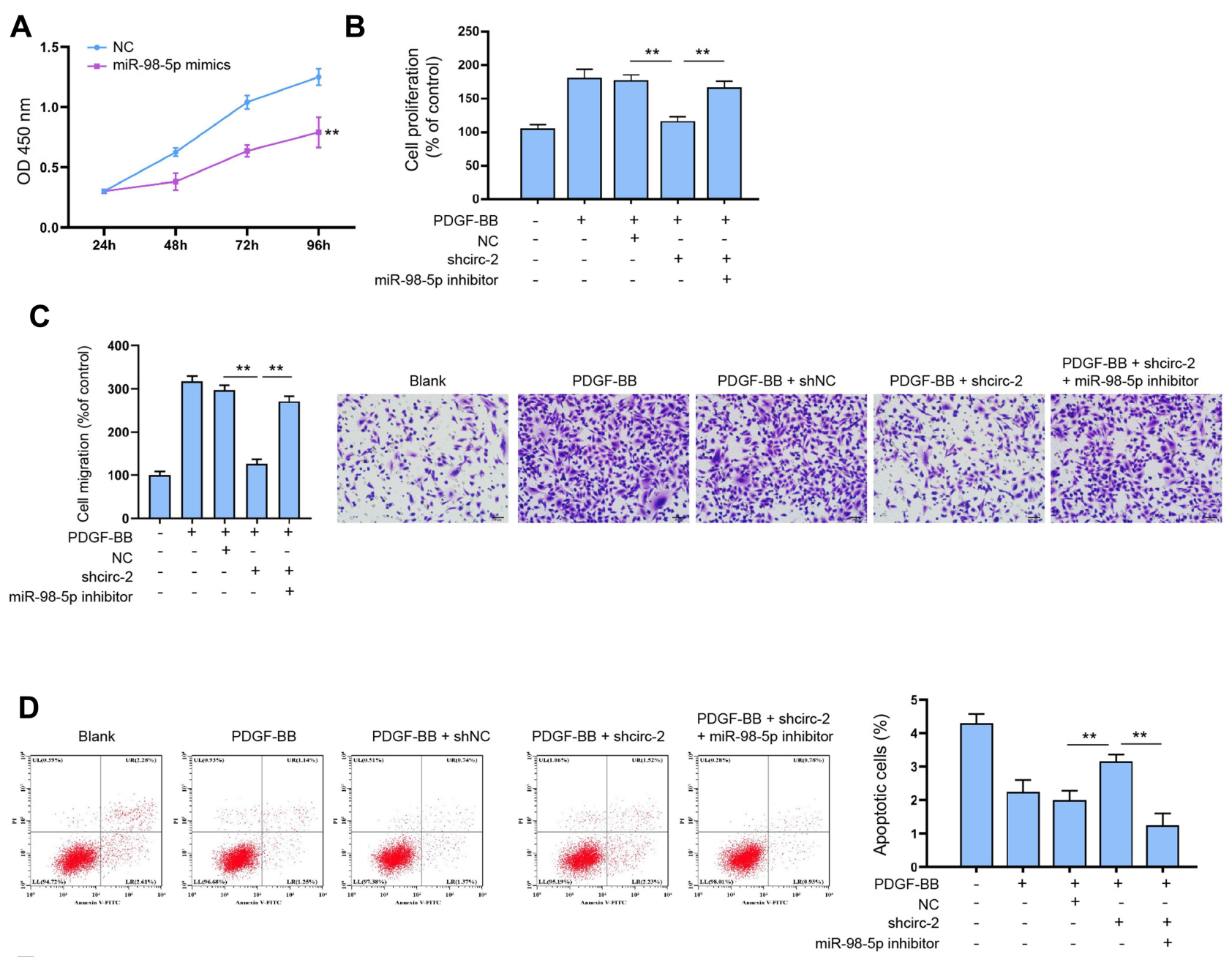

$\mathbf{E}$

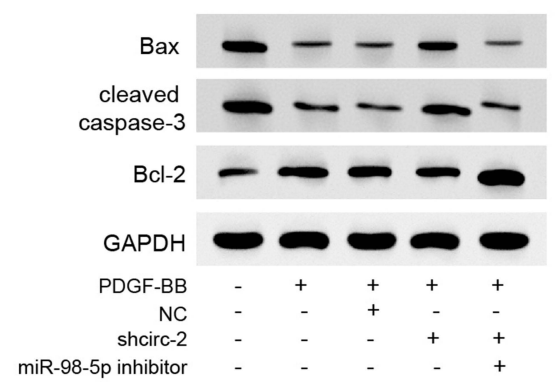

Figure 4 MiR-98-5p suppressed the PDGF-BB-stimulated proliferation and migration of ASMCs. (A) Viability of ASMCs after transfection with miR-98-5P mimics was determined by CCK-8. (B, C) CCK-8 assay and Transwell assay to determine viability and migration of ASMCs treated with PDGF-BB, shcircERBB2 and miR-98-5P inhibitors. Histogram showed the portion of viable or migrated cells. (D) Flow cytometry to detect cell migration. Histogram showed the portion of apoptotic cells. (E) The expression of Bax, cleaved caspase- 3 , and $\mathrm{Bcl}-2$ was measured by Western blot analysis. $* * p<0.01$.

Western blotting demonstrated that circERBB2 depletion downregulated the expression of IGF1R, and inhibition of miR-98-5p relived this effect (Figure $5 \mathrm{H}$ ). Subsequently, we demonstrated that IGFIR overexpression abolished the effects of miR-98-5p on PDGF-BB-stimulated ASMCs, manifested by recovered cell proliferation (Figure 6A), migration (Figure 6B) and apoptosis (Figure 6C and D).

\section{CircERBB2/miR-98-5p/IGFIR Axis} Regulated the Proliferation, Migration and Apoptosis of ASMCs

The above results have determined the interaction between miR-98-5p with circERBB2 and 3'UTR of IGF1R, and we next evaluated the existence of $\operatorname{circERBB2} / \mathrm{miR}-98-5 \mathrm{p} /$ 

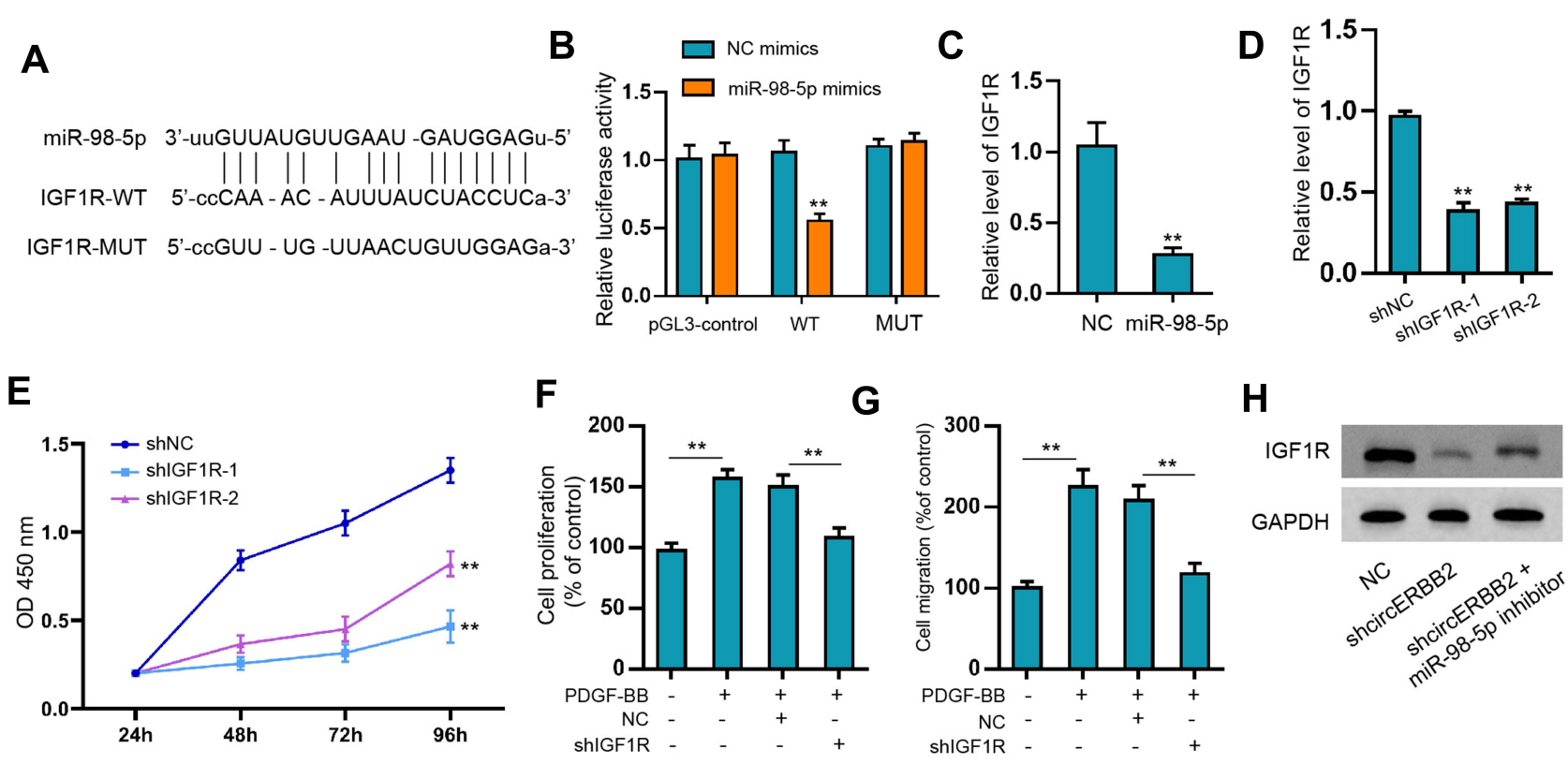

Figure 5 MiR-98-5p interacted with 3'UTR of IGFIR and IGFIR regulated PDGF-BB-stimulated proliferation and migration of ASMCs. (A) Predicted binding site of miR-98$5 p$ on 3'UTR of IGFIR. (B) Luciferase activity of IGFIR-WT and IGFIR-MUT after transfection with miR-98-5p mimics or NC. (C) QRT-PCR assay to measure the level of IGFIR in ASMCs after miR-98-5p transfection. (D) QRT-PCR assay to measure the level of IGFIR after transfection with shIGFIR-I, shIGFIR-2 or shNC. (E, F) CCK-8 assay to determine cell viability. (G) Transwell assay to detect cell migration. The histogram showed the portion of migrated cells. $(\mathbf{H})$ Western blotting assay to detect the expression of IGFIR. ${ }^{* *} p<0.0$ I.

$I G F 1 R$ regulatory axis in ASMCs. We transfected circERBB2 overexpressing vectors (circERBB2 OE), circERBB2 OE + miR-98-5p, or circERBB2 OE + miR98-5p + IGF1R OE in ASMCs, and then performed CCK8 , Transwell and flow cytometry. The results suggested that miR-98-5p significantly inhibited cell proliferation (Figure 7A), migration (Figure 7B) and upregulated apoptosis-induced by circERBB2 (Figure 7C and D), while the overexpression of IGF1R reversed these effects.

\section{Discussion}

As a chronic inflammatory respiratory disease, asthma has become a severe public problem that threatens the normal lives of human beings. ${ }^{2,3}$ Airway inflammation and remodeling are the most important processes in asthma, during which the abnormal proliferation and migration of ASMCs play critical roles. ${ }^{4,28,29}$ An increasing number of evidences have disclosed that various growth factors such as the platelet-derived growth factor (PDGF), fibroblast growth factor (FGF), transforming growth factor (TGF), and epidermal growth factor (EGF), are closely associated with airway remodeling and the severity of asthma. ${ }^{30}$ PDGF-BB is a member of the PDGF family and is reported to drive the switch of ASMCs to a highly proliferative and migratory phenotype, ${ }^{31}$ hence was applied in cell model to mimic ASMCs remodeling. In this work, we found elevated level of circERBB2 in patients with asthma, as well as in ASMCs after PDGF-BB treatment. These data preliminarily indicated the promoting role of circERBB2 and IGF1R in pathogenesis of asthma. As a newly defined circular RNA, circERBB2 was only reported as a cancer-related biomarker, ${ }^{20}$ yet its role during asthma was never defined. A recent next-generation sequencing study revealed aberrantly expressed circRNAs via using a murine asthma model. ${ }^{32}$ Circ_0005519 may be a potential biomarker for asthma and induce abnormal levels of IL- 6 and IL-13 in CD4 ${ }^{+}$ T cells through regulating let-7a-5p. ${ }^{33}$ Targeting circRNAs may become a novel therapeutic approach for asthma therapy. Importantly, the correlation of circERBB2 with asthma has not been reported previously. We disclosed that knockdown of circERBB2 suppressed the PDGF-BBstimulated proliferation and migration of ASMCs under PDGF-BB treatment, along with elevated cell apoptosis and impaired secretion of inflammatory factors. These results demonstrate that circERBB2 play a critical role during PDGF-BB-stimulated proliferation and migration of ASMCs.

MiRNAs belong to non-coding RNAs with a short length of around 20 nucleotides and disturb gene expression by targeting the 3 'UTR regions of mRNAs, consequently leading to mRNA degradation or inhibited 

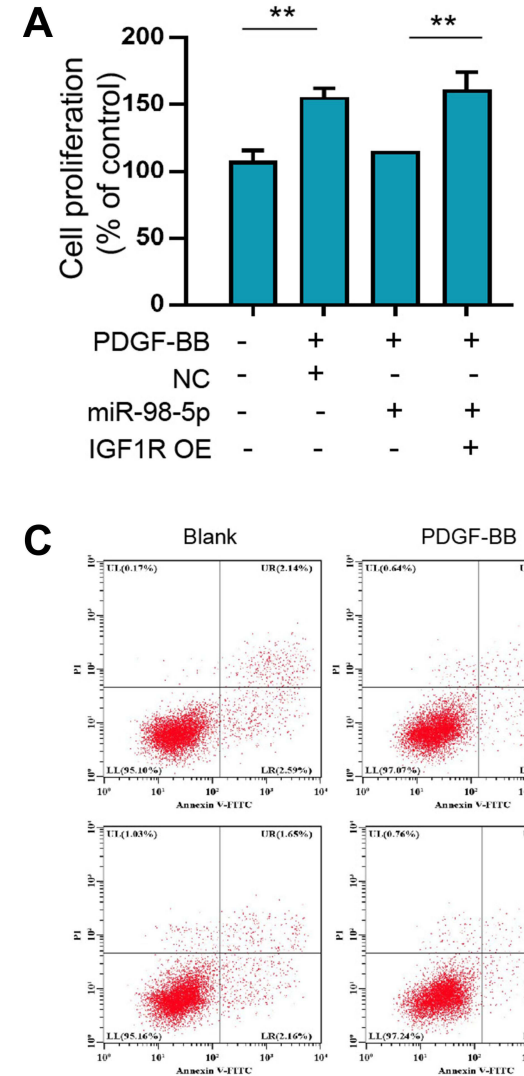

PDGF-BB + miR-98-5p
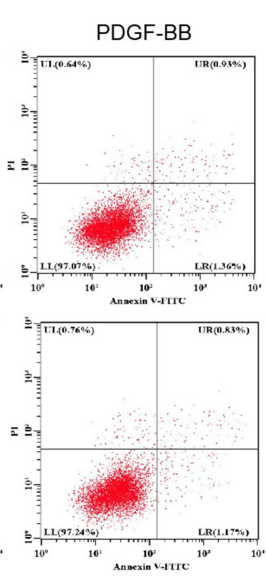

PDGF-BB +

miR-98-5p + IGF1R OE
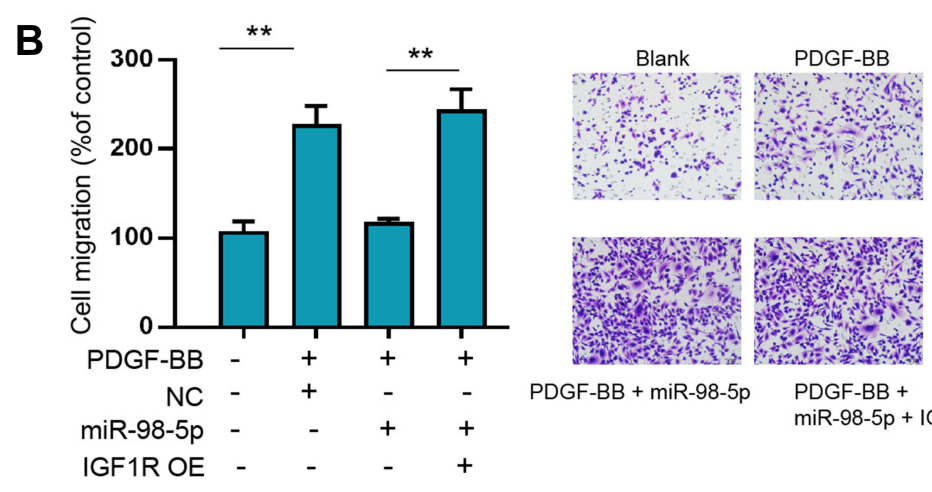

PDGF-BB + miR-98-5p

PDGF-BB +

miR-98-5p + IGF1R OE

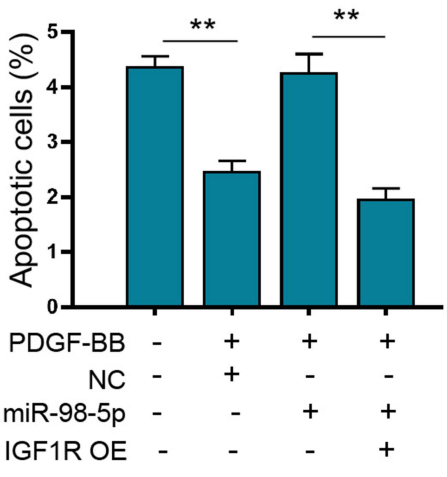

D

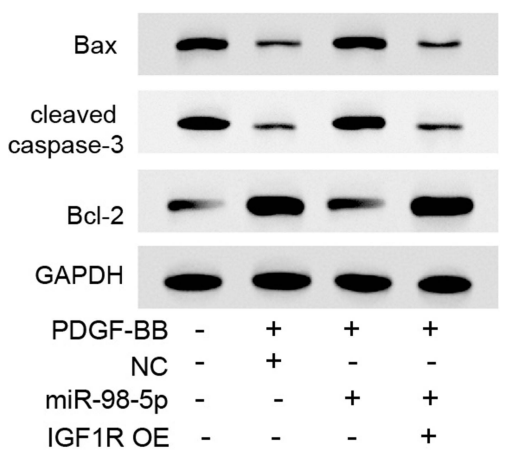

Figure 6 IGFIR mediated miR-98-5p-suppressed proliferation and migration of ASMCs. ASMCs were treated with PDGF-BB, and transfected with miR-98-5p, IGFIR overexpressing vector (IGFIR OE) or negative controls. Then the proliferation (A), migration (B), and apoptosis (C) of ASMCs were determined by CCK-8, Transwell, and flow cytometry assay, respectively. (D) The expression of Bax, cleaved caspase-3, and $\mathrm{Bcl}-2$ was measured by Western blot analysis. $* * p<0.0 \mathrm{I}$.

translation. $^{22}$ Substantial studies have indicated the involvement of miRNAs in various critical biological processes including proliferation, migration and inflammatory responses, and in multifactorial diseases, including asthma. ${ }^{34}$ MiR-98-5p was reported to participate in the progression of several diseases. For example, miR-98-5p targeted Gab2 to regulate proliferation and migration of breast cancer cells. ${ }^{35}$ During diabetic nephropathy, miR98-5p inhibited epithelial to mesenchymal transition and renal fibrosis via targeting HMGB2. ${ }^{36}$ Moreover, Du and colleagues proposed that miR-98-5p was a potential diagnostic biomarker for bronchial asthma in children, and affected the level of IL-13 in serum. ${ }^{24}$ Importantly, the relationship between miR-98-5p and circERBB2 has not been reported previously. Our data showed an elevated level of miR-98-5p in PDGF-BB-stimulated cell model, as well as a direct interaction between circERBB2 with miR-98-5p. These data suggest that miR-98-5p is a direct target of circERBB2.
The regulatory role of IGF1R during pathogenesis in asthma has been previously reported. Upregulated level of IGF1R was found in bronchoalveolar lavage cells from asthma patients. ${ }^{14} \mathrm{~A}$ recent study revealed that IGF1R deficient mice exhibited reduced allergic airway inflammation. ${ }^{37}$ On the other hand, miRNAs targeting IGF1R were also reported in regulation of asthma. For example, miR-223 was discovered to cause phenotype switch of ASMCs and remodeling of extracellular matrix, through targeting IGF1R. ${ }^{38}$ Our work presented that miR-98-5p targeted the mRNA of IGF1R to affect the ASMCs remodeling, and mediated the function of circERBB2 under stimulation of PDGF-BB. It suggests that targeting IGF1R is one of the mechanisms by which circERBB2/miR-98-5p axis regulates asthma and other potential mechanisms should be explored by more investigations. The above mentioned experimental outcomes tempted us to propose that circERBB2/miR-98$5 \mathrm{p} / \mathrm{IGF} 1 \mathrm{R}$ axis functioned during ASMCs remodeling under PDGF-BB stimulation. 

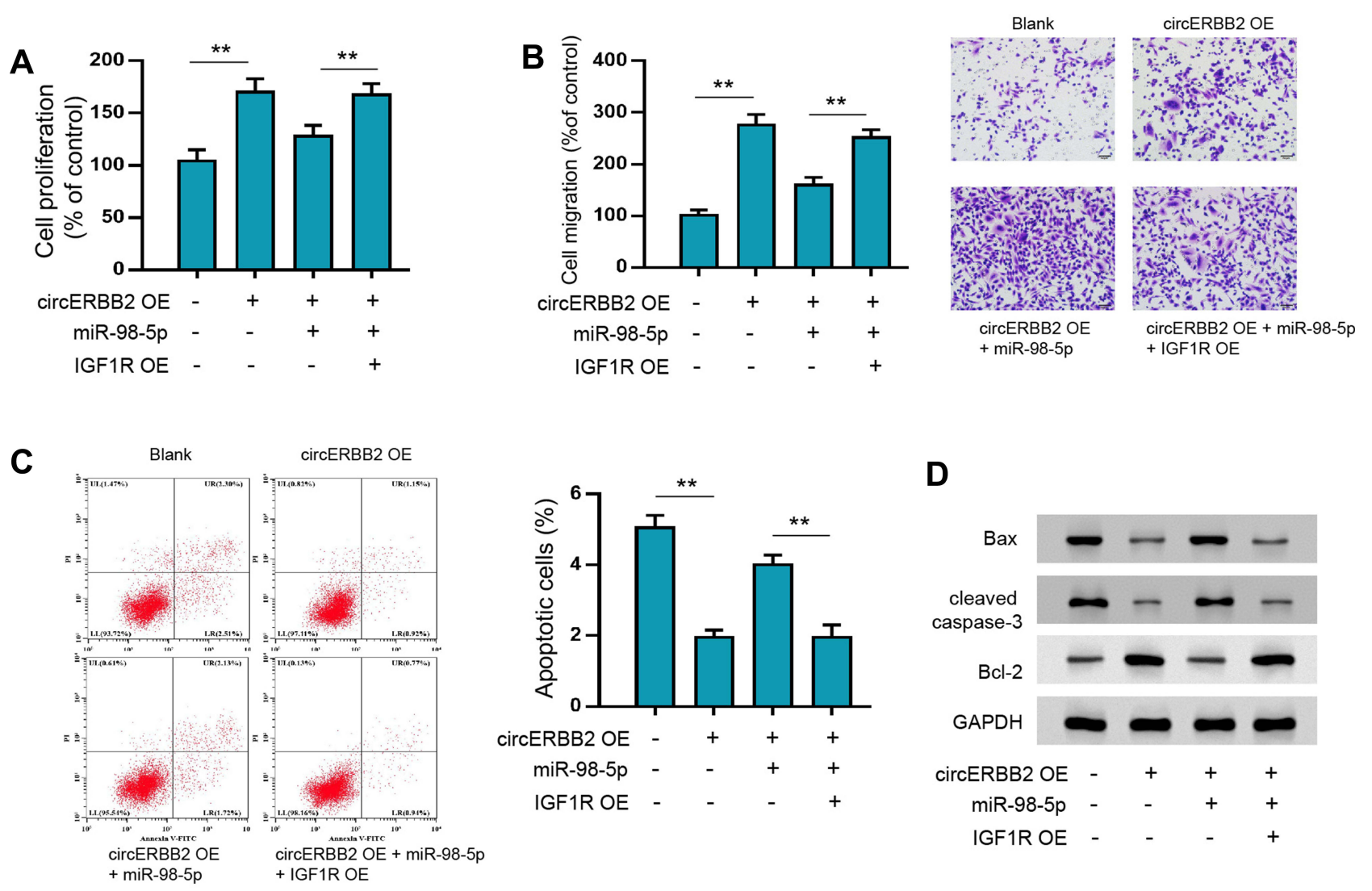

Figure 7 CircERBB2/miR-98-5p/IGFIR axis regulated the proliferation, migration and apoptosis of ASMCs. ASMCs were transfected with circERBB2 overexpressing vectors (circERBB2 OE), circERBB2 OE + miR-98-5p, or circERBB2 OE + miR-98-5p + IGFIR OE. Then, cell proliferation (A), migration (B), and apoptosis (C) of ASMCs were determined by CCK-8, Transwell, and flow cytometry assay, respectively. (D) The expression of Bax, cleaved caspase-3, and Bcl-2 was measured by Western blot analysis. $* * p<0.01$.

\section{Conclusions}

In summary, our work disclosed the promoting role of circERBB2 during PDGF-BB stimulated ASMCs remodeling and inflammation, and knockdown of circERBB2 suppressed PDGF-BB-caused proliferation, migration and inflammatory response of ASMCs through sponging miR98-5p and suppression of IGF1R signaling. These data presented circERBB2 as a novel target for therapy of asthma.

\section{Disclosure}

The authors report no conflicts of interest in this work.

\section{References}

1. Lemanske RF Jr., Busse WW. Asthma. JAMA. 1997;278:1855-1873.

2. Mims JW. Asthma: definitions and pathophysiology. Int Forum Allergy Rhinol. 2015;5(Suppl 1):S2-6.

3. Sockrider M, Fussner L. WhatIs Asthma? Am J Respir Crit Care Med. 2020;202:P25-P6.

4. Bousquet J, Mantzouranis E, Cruz AA, et al. Uniform definition of asthma severity, control, and exacerbations: document presented for the World Health Organization Consultation on Severe Asthma. J Allergy Clin Immunol. 2010;126:926-938.
5. Poon AH, Hamid Q. Severe asthma: have we made progress? Ann Am Thorac Soc. 2016;13(Suppl 1):S68-77.

6. Castillo JR, Peters SP, Busse WW. Asthma exacerbations: pathogenesis, prevention, and treatment. J Allergy Clin Immunol Pract. 2017;5:918-927.

7. Hartley R, Berair R, Brightling CE. Severe asthma: novel advances in the pathogenesis and therapy. Pol Arch Med Wewn. 2014;12 $4: 247-254$.

8. Lin L, Li Q, Hao W, Zhang Y, Zhao L, Han W. Upregulation of LncRNA Malat1 induced proliferation and migration of airway smooth muscle cells via miR-150-eIF4E/Akt signaling. Front Physiol. 2019;10:1337.

9. Pang X, Qiao J. Galectin-1 inhibits PDGF-BB-induced proliferation and migration of airway smooth muscle cells through the inactivation of PI3K/Akt signaling pathway. Biosci Rep. 2020;40: BSR20193899.

10. Seidel P, Goulet S, Hostettler K, Tamm M, Roth M. DMF inhibits PDGF-BB induced airway smooth muscle cell proliferation through induction of heme-oxygenase-1. Respir Res. 2010;11: 145 .

11. Girnita L, Worrall C, Takahashi S, Seregard S, Girnita A. Something old, something new and something borrowed: emerging paradigm of insulin-like growth factor type 1 receptor (IGF-1R) signaling regulation. Cell Mol Life Sci. 2014;71:2403-2427.

12. Hoshino M, Nakamura Y, Sim JJ, et al. Inhaled corticosteroid reduced lamina reticularis of the basement membrane by modulation of insulin-like growth factor (IGF)-I expression in bronchial asthma. Clin Exp Allergy. 1998;28:568-577. 
13. Veraldi KL, Gibson BT, Yasuoka H, et al. Role of insulin-like growth factor binding protein-3 in allergic airway remodeling. Am J Respir Crit Care Med. 2009;180:611-617.

14. Esnault S, Kelly EA, Schwantes EA, et al. Identification of genes expressed by human airway eosinophils after an in vivo allergen challenge. PLoS One. 2013;8:e67560.

15. Pineiro-Hermida S, Alfaro-Arnedo E, Gregory JA, et al. Characterization of the acute inflammatory profile and resolution of airway inflammation after Igflr-gene targeting in a murine model of HDM-induced asthma. PLoS One. 2017;12:e0190159.

16. Salzman J, Gawad C, Wang PL, Lacayo N, Brown PO. Circular RNAs are the predominant transcript isoform from hundreds of human genes in diverse cell types. PLoS One. 2012;7:e30733.

17. Vo JN, Cieslik M, Zhang Y, et al. The Landscape of Circular RNA in Cancer. Cell. 2019;176:869-81 e13.

18. Ferreira D, Soares M, Correia J, Adega F, Ferreira F, Chaves R. Assessment of ERBB2 and TOP2alpha gene status and expression profile in feline mammary tumors: findings and guidelines. Aging. 2019;11:4688-4705.

19. Krishnamurti U, Silverman JF. HER2 in breast cancer: a review and update. Adv Anat Pathol. 2014;21:100-107.

20. Huang X, He M, Huang S, et al. Circular RNA circERBB2 promotes gallbladder cancer progression by regulating PA2G4-dependent rDNA transcription. Mol Cancer. 2019;18:166.

21. Nanishi K, Konishi H, Shoda K, et al. Circulating circERBB2 as a potential prognostic biomarker for gastric cancer: an investigative study. Cancer Sci. 2020;111:4177-4186.

22. Bartel DP. MicroRNAs: genomics, biogenesis, mechanism, and function. Cell. 2004;116:281-297.

23. Gao J, Ding C, Zhou J, et al. Propofol suppresses lung cancer tumorigenesis by modulating the circ-ERBB2/miR-7-5p/FOXM1 axis. Thorac Cancer. 2021;12:824-834.

24. Du J, Wu H, Wu Y. MiR-98-5p may be a biomarker for screening bronchial asthma in children by targeting IL-13. Clin Lab. 2019;65:1.

25. Busse WW, Wanner A, Adams K, et al. Investigative bronchoprovocation and bronchoscopy in airway diseases. Am J Respir Crit Care Med. 2005;172:807-816.

26. Yick CY, Zwinderman AH, Kunst PW, et al. Glucocorticoid-induced changes in gene expression of airway smooth muscle in patients with asthma. Am J Respir Crit Care Med. 2013;187:1076-1084.
27. Yick CY, Zwinderman AH, Kunst PW, et al. Gene expression profiling of laser microdissected airway smooth muscle tissue in asthma and atopy. Allergy. 2014;69:1233-1240.

28. Bentley JK, Hershenson MB. Airway smooth muscle growth in asthma: proliferation, hypertrophy, and migration. Proc Am Thorac Soc. 2008;5:89-96.

29. Prakash YS. Airway smooth muscle in airway reactivity and remodeling: what have we learned? Am J Physiol Lung Cell Mol Physiol. 2013;305:L912-33.

30. Hoshino M, Takahashi M, Aoike N. Expression of vascular endothelial growth factor, basic fibroblast growth factor, and angiogenin immunoreactivity in asthmatic airways and its relationship to angiogenesis. J Allergy Clin Immunol. 2001;107:295-301.

31. Halayko AJ, Tran T, Gosens R. Phenotype and functional plasticity of airway smooth muscle: role of caveolae and caveolins. Proc Am Thorac Soc. 2008;5:80-88.

32. Bao H, Zhou Q, Li Q, et al. Differentially expressed circular RNAs in a murine asthma model. Mol Med Rep. 2020;22:5412-5422.

33. Huang Z, Cao Y, Zhou M, et al. Hsa_circ_0005519 increases IL-13/ IL-6 by regulating hsa-let-7a-5p in $\mathrm{CD}^{-}(+\overline{+}) \mathrm{T}$ cells to affect asthma. Clin Exp Allergy. 2019;49:1116-1127.

34. Rupaimoole R, Slack FJ. MicroRNA therapeutics: towards a new era for the management of cancer and other diseases. Nat Rev Drug Discov. 2017;16:203-222.

35. Shi XY, Wang H, Wang W, Gu YH. MiR-98-5p regulates proliferation and metastasis of MCF-7 breast cancer cells by targeting Gab2. Eur Rev Med Pharmacol Sci. 2020;24:10914.

36. Zhu Y, Xu J, Liang W, et al. miR-98-5p alleviated epithelial-tomesenchymal transition and renal fibrosis via targeting Hmga2 in diabetic nephropathy. Int J Endocrinol. 2019;2019:4946181.

37. Pineiro-Hermida S, Lopez IP, Alfaro-Arnedo E, et al. IGF1R deficiency attenuates acute inflammatory response in a bleomycin-induced lung injury mouse model. Sci Rep. 2017;7:4290.

38. Liu D, Pan J, Zhao D, Liu F. MicroRNA-223 inhibits deposition of the extracellular matrix by airway smooth muscle cells through targeting IGF-1R in the PI3K/Akt pathway. Am J Transl Res. 2018;10:744-752.

\section{Publish your work in this journal}

The Journal of Asthma and Allergy is an international, peer-reviewed open-access journal publishing original research, reports, editorials and commentaries on the following topics: Asthma; Pulmonary physiology; Asthma related clinical health; Clinical immunology and the immunological basis of disease; Pharmacological interventions and new therapies. The manuscript management system is completely online and includes a very quick and fair peer-review system, which is all easy to use. Visit http://www.dovepress.com/testimonials.php to read real quotes from published authors. 